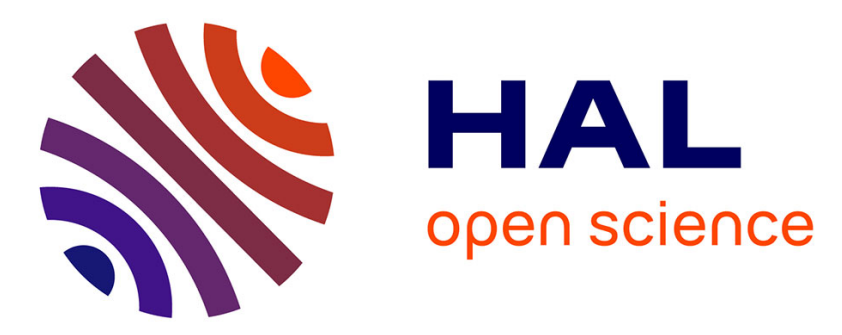

\title{
Analyse angulaire de la fluorescence du fer dans une multicouche périodique $\mathrm{Fe} / \mathrm{C}$
}

Jean-Pierre Chauvineau, Françoise Bridou

\section{To cite this version:}

Jean-Pierre Chauvineau, Françoise Bridou. Analyse angulaire de la fluorescence du fer dans une multicouche périodique Fe/C. Journal de Physique IV Proceedings, 1996, 06 (C7), pp.C7-53-C7-64. 10.1051/jp4:1996707 . jpa-00254495

\section{HAL Id: jpa-00254495 https://hal.science/jpa-00254495}

Submitted on 1 Jan 1996

HAL is a multi-disciplinary open access archive for the deposit and dissemination of scientific research documents, whether they are published or not. The documents may come from teaching and research institutions in France or abroad, or from public or private research centers.
L'archive ouverte pluridisciplinaire HAL, est destinée au dépôt et à la diffusion de documents scientifiques de niveau recherche, publiés ou non, émanant des établissements d'enseignement et de recherche français ou étrangers, des laboratoires publics ou privés. 


\title{
Analyse angulaire de la fluorescence du fer dans une multicouche périodique $\mathrm{Fe} / \mathrm{C}$
}

\author{
J.P. Chauvineau et F. Bridou \\ Institut d'Optique, URA 14 du CNRS, Centre Universitaire, BP. 147, 91403 Orsay cedex, France
}

\begin{abstract}
Résumé: On a mesuré la variation angulaire de l'intensité du rayonnement de fluorescence K $\alpha$ du fer émis sous émergence rasante par une multicouche périodique $\mathrm{Fe} / \mathrm{C}$. On observe une forte modulation de l'intensité émise au voisinage de l'angle d'émergence correspondant à la direction de diffraction de Bragg du rayonnement caractéristique du fer par le milieu stratifié périodique. Cette modulation est semblable aux lignes de Kossel observables sur les diagrammes de fluorescence de monocristaux; sa position et sa forme sont bien reproduites par un calcul basé sur l'utilisation du théorème de réciprocité et du formalisme de calcul des propriétés optiques des couches minces. La multicouche a ensuite été irradiée avec un rayonnement quasi-monochromatique (raies $K \alpha$ du cuivre) sous incidence rasante au voisinage d'un angle de Bragg afin de moduler l'intensité du champ électrique excitateur en fonction de la profondeur. On met en évidence les variations de l'intensité du rayonnement de fluorescence du fer en fonction de l'angle d'incidence du rayonnement excitateur.
\end{abstract}

\section{INTRODUCTION.}

Les multicouches synthétiques périodiques sont maintenant d'un usage bien établi pour la réalisation de composants optiques tels que des miroirs sélectifs fonctionnant en incidence normale dans le domaine du rayonnement X-UV ou en incidence rasante pour les rayons X [1]. Dans la plupart des cas, elles sont faites avec deux matériaux dont les indices optiques en X-UV sont les plus différents possible pour obtenir un pouvoir réflecteur important; pratiquement, on associe un métal relativement dense comme matériau de "haut indice" à un élément léger $(\mathrm{B}, \mathrm{C}, \mathrm{Si}, . .$.$) comme matériau de "bas indice". L'épaisseur de chaque couche$ élémentaire est typiquement de l'ordre de un à quelques nanomètres, et l'efficacité de ces traitements repose en grande partie sur la qualité des interfaces dont la largeur doit rester faible par rapport à la longueur d'onde d'utilisation, ce qui nécessite une très bonne planété et l'absence d'interdiffusion entre les matériaux juxtaposés. La mesure de la réflectivité en rayons $X$ en fonction de l'angle d'attaque $\theta$, sous incidence rasante est d'ailleurs une méthode largement utilisée pour caractériser la structure géométrique des multicouches, car l'analyse des courbes $R(\theta)$ permet d'obtenir le profil de la densité électronique moyenne dans un plan parallèle aux interfaces en fonction de la profondeur, et par conséquent de déterminer la valeur de la période et la largeur moyenne des zones interfaciales [2]. Cependant, cette méthode repose sur la simulation des courbes expérimentales à partir d'un modèle de profil d'indice et ne fournit pas toujours une solution unique en raison des incertitudes de mesure et des imperfections de l'échantillon qui ne sont pas prises en compte par le modèle. Il est donc intéressant d'associer à cette technique des méthodes d'analyse complémentaires susceptibles de lever d'éventuelles ambiguïtés, et parmi les méthodes envisageables l'étude du rayonnement de fluorescence $X$ possède, en commun avec la réflectométrie $X$ rasants, l'avantage d'être non-destructive.

L'intérêt pratique d'étudier la fluorescence pour faire de l'analyse élémentaire de couches superficielles a été démontré par plusieurs travaux dans lesquels on a mis à profit la variation de la profondeur de pénétration [3] et la modulation d'intensité du rayonnement primaire dans l'échantillon en fonction de l'angle en incidence rasante [4] pour établir des profils de concentration au voisinage de la surface. Notre objectif est de montrer que l'étude de la distribution angulaire du rayonnement de fluorescence des multicouches périodiques peut constituer une méthode de caractérisation non destructive capable de déterminer le profil de concentration en profondeur d'un élément donné, notamment au niveau des interfaces où ce profil influe considérablement sur les performances optiques en X-UV de l'empilement.

Le rayonnement caractéristique émis dans un monocristal par fluorescence d'un élément peut être diffracté par le cristal lui-même; ceci donne lieu à des modulations dans la distribution angulaire de l'intensité du rayonnement de fluorescence divergent issu du cristal et conduit à l'observation d'un diagramme formé de 
lignes appelées "lignes de Kossel" sur un film plan placé a proximité [5]. Des effets de l'interférence du rayonnement de fluorescence dans des matériaux stratifiés ont également été observés $[6,7,8]$. Nous avons montré récemment qu'un milieu stratifié périodique tel qu'une multicouche destinée à réfléchir le rayonnement $\mathrm{X}-\mathrm{UV}$, constituée par un empilement périodique de strates alternées de très faible épaisseur peut induire des effets semblables avec le rayonnement de fluorescence de chacun de ses éléments constitutifs [9].

\section{DISPOSITIFS EXPERIMENTAUX.}

\subsection{Multicouches.}

Les multicouches $\mathrm{Fe} / \mathrm{C}$ sont fabriquées par pulvérisation ionique sous atmosphère d'argon $\left(2.10^{-4} \mathrm{mbar}\right)$. L'épaisseur des couches élémentaires est contrôlée par réflectométrie de rayons X mous pendant le dépôt. La période est voisine de $5 \mathrm{~nm}$, les épaisseurs des couches de fer et de carbone étant sensiblement égales. Le nombre de périodes dans l'empilement est de 24. Le substrat - un disque de silicium monocristallin dont la surface polie a une rugosité très faible - est maintenu à la température ambiante pendant le dépôt des couches.

\section{Tableau I}

\begin{tabular}{|c|r|r|r|r|}
\hline & Epaisseur $(\AA)$ & Rugosité $(\AA)$ & $(1-n)\left(\times 10^{6}\right)$ & Extinction $\left(\times 10^{6}\right)$ \\
\hline Substrat & 0 & 2.07 & 6.75 & 0.166 \\
\cline { 2 - 5 }$F_{\theta}$ & 28 & 4.5 & 18.074 & 2.996 \\
\hline$C$ & 25.58 & 2 & 7.64 & 0.999 \\
\hline
\end{tabular}

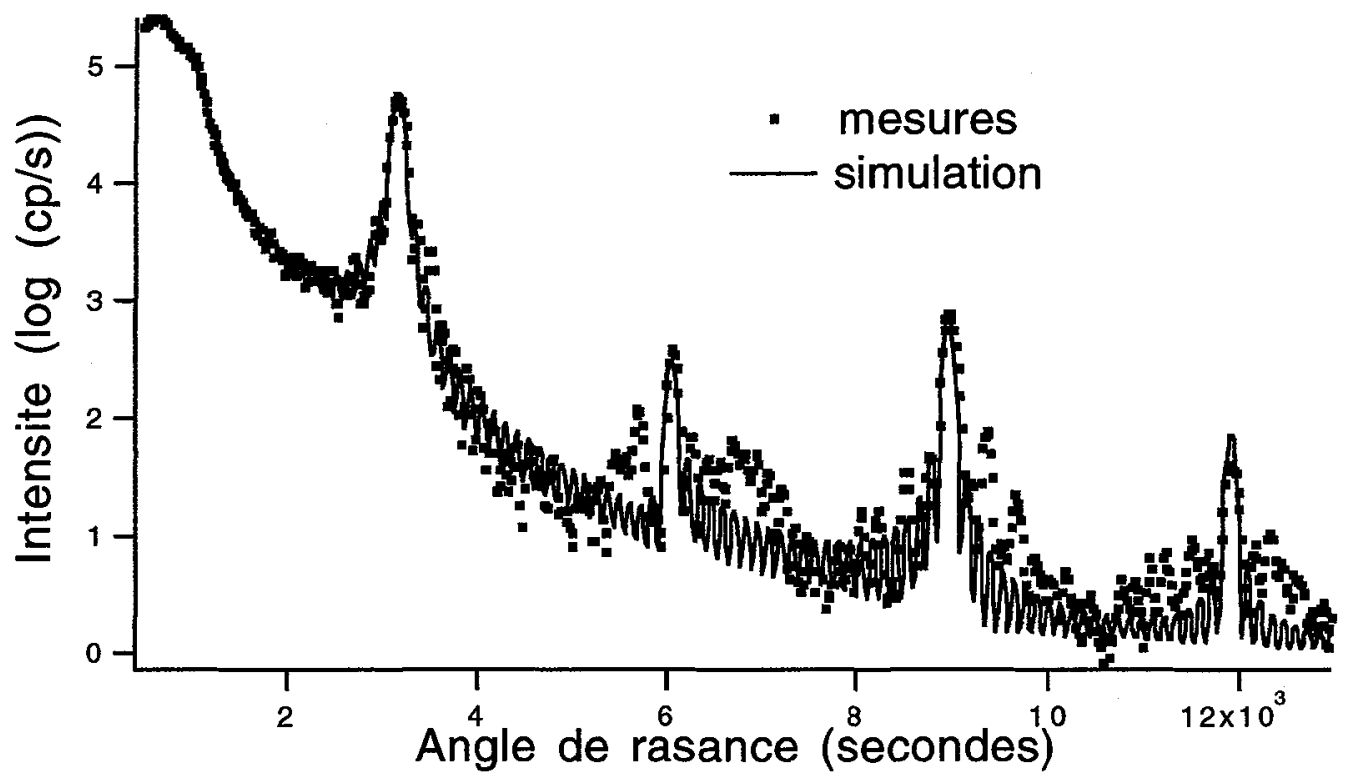

Figure 1: Analyse d'une multicouche comprenant 24 bicouches $\mathrm{Fe} / \mathrm{C}$ par reflectométrie de rayons $\mathrm{X}$ rasants $(\mathrm{E}=8048 \mathrm{eV}) ; \mathrm{la}$ courbe thérique (trait continu) est calculée à partir des valeurs du tableau $\mathrm{I}$.

L'analyse des multicouches par réflectométrie de rayons $X$ rasants à $0,154 \mathrm{~nm}$ fait apparaître les pics de Bragg caractéristiques de leur structure périodique dans la direction perpendiculaire au substrat, alors qu'aucune structure particulière n'est observable en diffraction $\mathrm{X}$ à grand angle, indiquant le caractère très désordonné des matériaux à l'échelle atomique dans les couches ultra-minces de fer et de carbone. 
La simulation de la courbe de réflectivité expérimentale (Figure 1) est faite à partir d'un calcul dans lequel les paramètres ajustés par une méthode d'essais et erreurs [10] sont les épaisseurs moyennes des couches, leur indice et la largeur moyenne des interfaces dont l'influence sur la réflectivité est évaluée en l'assimilant à une rugosité [2]. Les résultats sont reportés dans le tableau $I$.

L'écart résiduel entre les points expérimentaux et la réflectivité calculée apparaît surtout entre les pics de Bragg et provient des fluctuations de l'épaisseur des couches autour des valeurs moyennes utilisées dans le modèle. Les défauts de planéité du substrat entrainent aussi une déformation de la courbe de réflexion spéculaire observable dans la région de réflexion quasi-totale, sous incidence très rasante [11].

Les indices obtenus s'écartent légèrement des valeurs tabulées pour le fer et le carbone dans le sens d'un accroissement pour le carbone et d'une diminution pour le fer. On constate par ailleurs une dissymétrie de la largeur moyenne des deux types d'interfaces correspondant respectivement à $\mathrm{Fe} / \mathrm{C}$ et $\mathrm{C} / \mathrm{Fe}$. Ces résultats suggèrent la possibilité d'une interdiffusion des deux matériaux; la vérification de cette hypothèse nécessite de faire appel à une méthode de caractérisation telle que l'analyse par fluorescence X, capable de déterminer la composition d'un milieu stratifié en fonction de la profondeur.

\subsection{Fluorescence du fer.}

L'étude expérimentale du rayonnement de fluorescence du fer à $6400 \mathrm{eV}$ (raies Ko1,2) est faite à l'aide d'un diffractomètre $X$ classique équipé d'un tube à anode de cuivre.

Pour cette raie du fer, l'angle d'émergence correspondant à la réflexion de Bragg du premier ordre dans l'empilement est voisin de $1^{\circ}$.

\section{Détecteur (1) (Film ou compteur)}

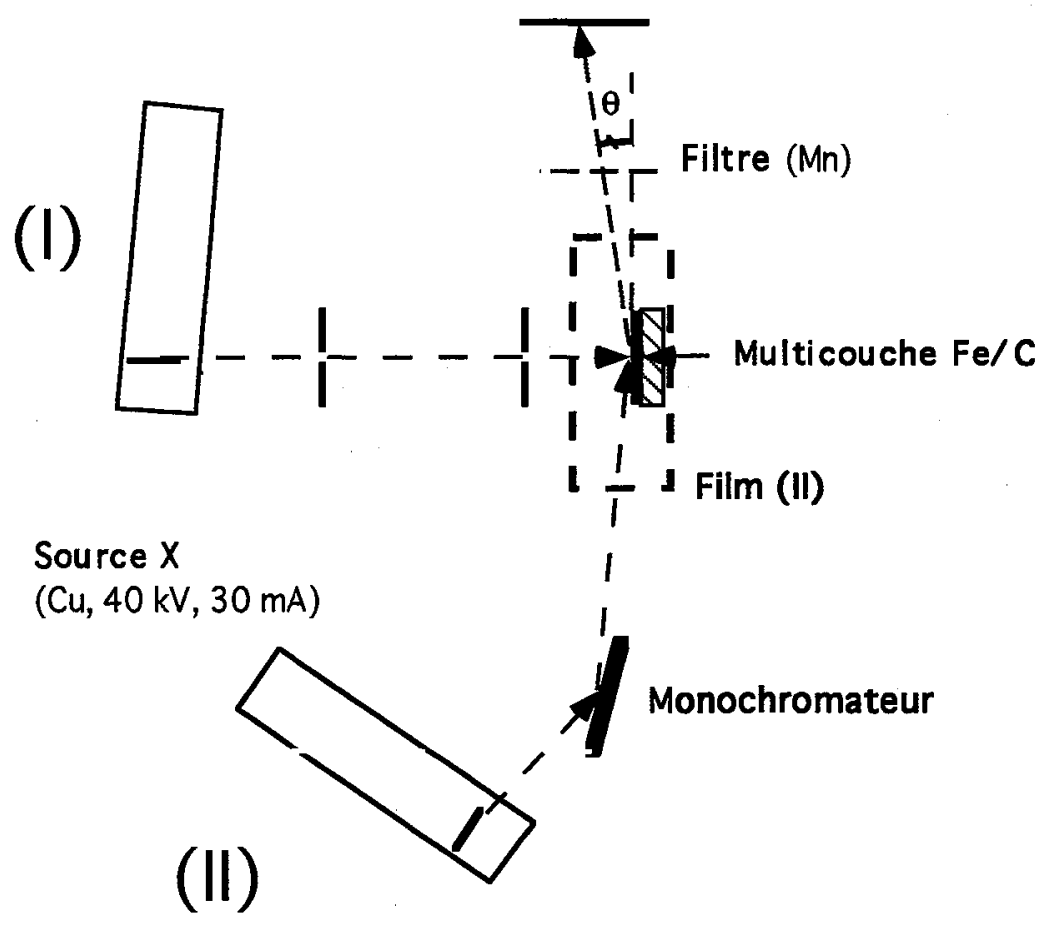

Figure 2: Dispositif expérimental de mesure de l'intensité du rayonnement de fluorescence $\mathrm{K} \alpha$ du fer en fonction de l'angle d'émergence rasante $\theta$.

I: éclairage sous incidence normale

II: éclairage sous incidence rasante

Dans la première série de mesures destinées à mettre en évidence la variation de l'intensité de fluorescence en fonction de l'angle d'émergence, la multicouche est éclairée sous incidence normale par le rayonnement 
issu du tube, et la partie du rayonnement de fluorescence émise au voisinage de l'émergence rasante est enregistrée sur un film plan perpendiculaire au substrat, ou mesurée en fonction de l'angle d'émergence avec un compteur proportionnel placé derrière une fente d'analyse. Dans ce dernier cas, un filltre de manganèse ou un monochromateur en graphite peuvent être interposés entre l'échantillon et le détecteur pour réduire l'intensité du rayonnement $K \beta$ du fer par rapport au rayonnement $K \alpha$ (Figure 2). La durée d'exposition du film est de l'ordre de 4 heures, tandis que le temps de comptage avec le compteur proportionnel est de $100 \mathrm{~s}$ par point.

Dans la deuxième configuration, l'échantillon est éclairé sous incidence rasante et en rayonnement monochromatique pour pouvoir moduler l'intensité du champ excitateur dans la multicouche. On sélectionne les raies $\mathrm{K} \alpha$ du cuivre soit grossièrement par un filtre en nickel qui absorbe dans une bande spectrale comprenant les raies $K \beta$, soit par un monochromateur en graphite. Le rayonnement de fluorescence est enregistré sur un film photographique placé parallèlement au plan d'incidence pour éviter de recevoir le rayonnement primaire diffusé principalement par l'échantillon au voisinage de la direction de réflexion spéculaire. Les temps d'exposition varient de quelques heures à plusieurs dizaines d'heures suivant l'ouverture du faisceau incident.

\section{MODELISATION.}

On suppose que la concentration ci des éléments constituant la multicouche $(i=\mathrm{Fe}$ ou $\mathrm{C}$ ) ne dépend que de la profondeur $\mathrm{z}$ dans l'empilement. Si la source de rayonnement primaire est suffisamment éloignée de l'échantillon, on peut considérer que les ondes électromagnétiques incidentes sont des ondes planes et, en conséquence de la stratification du milieu, génèrent dans la multicouche un champ électromagnétique excitateur dont l'intensité $\left|E_{e x}\right|^{2}$ dépend de la profondeur $\mathrm{z}$ et de l'angle d'incidence $\theta_{i}$. Ce champ peut être calculé pour un rayonnement monochromatique en utilisant le formalisme récurrent qui sert à la détermination des propriétés optiques des empilements à partir des constantes optiques et des épaisseurs des couches [12]. L'intensité du rayonnement de fluorescence émis à une profondeur $z$ par l'élément $i$ est proportionnelle au produit $c_{i}(z) . \mid E_{e x}\left(\theta_{i}, z\right)^{2}$, en l'absence d'effet de fluorescence secondaire. Le coefficient de proportionnalité est le rendement de fluorescence qui dépend de la longueur d'onde du rayonnement excitateur.

La seconde étape du calcul consiste à déterminer l'intensité de fluorescence émergeant de l'échantillon sous un angle donné, émis à partir d'une source de rayonnement située à la profondeur z. Ce calcul est réalisé en appliquant le théorème de réciprocité d'Helmoltz [13] selon la méthode utilisée par Laue [14] qui a permis l'interprétation des diagrammes de Kossel. D'après ce théorème, le champ électrique cré à l'entrée du détecteur par une source dipolaire située dans un milieu donné a la même valeur que celui qui serait créé au point où se trouve la source par un dipôle identique placé à l'entrée du détecteur. La distance entre l'échantillon et le détecteur étant très grande par rapport à l'épaisseur des couches, on peut calculer ce dernier champ électrique $\mathrm{E}_{\mathrm{d}}$ en tout point de l'empilement en supposant que la source fictive qui rayonne à la fréquence caractéristique de l'élément considéré est située à l'infini dans la direction de détection et en utilisant le même formalisme que pour le calcul du champ excitateur . Compte-tenu de la géométrie du système, ce champ dépend de la profondeur $\mathrm{z}$ dans la multicouche et de l'angle d'émergence $\theta_{\mathrm{e}}$. L'intensité du rayonnement de fluorescence de l'élément i mesurée dans la direction $\theta_{\mathrm{e}}$ est en définitive proportionnelle à:

$$
c_{i}(z) \cdot\left|E_{e x}\left(\theta_{i}, z\right)\right|^{2} \cdot\left|E_{d}\left(\theta_{e}, z\right)\right|^{2}
$$

Dans la suite, les calculs sont faits en considérant le rayonnement $K \alpha$ du cuivre à $0,154 \mathrm{~nm}$ pour le champ excitateur et le rayonnement de fluorescence $\mathrm{K} \alpha$ du fer à $0,1936 \mathrm{~nm}$. Les épaisseurs retenues pour les couches de chacun des deux matériaux sont les valeurs moyennes obtenues par analyse de la courbe de réflectivité en $\mathrm{X}$ rasants. Dans un empilement supposé parfait la concentration $\mathrm{ci}(\mathrm{z})$ vaut 1 dans les couches de l'élément $i$ et 0 dans celles de l'autre élément et les constantes optiques utilisées sont extraites de tables publiées pour les matériaux massifs[15]. Pour mieux représenter la multicouche réelle, on peut utiliser les valeurs d'indices fournies par la simulation de la courbe de réflectivité (Tableau I) pour calculer le champ excitateur et pour déterminer un nouveau profil de concentration en supposant que les différences d'indice observées par rapport aux valeurs tabulées sont dues à une interdiffusion des matériaux, puis recalculer les constantes optiques correspondantes pour la raie de fluorescence du fer. Cependant, cette méthode implique de faire des hypothèses sur la densité des matériaux en couches ultra-minces. En définitive, la meilleure solution possible pour le profil de concentration devra être déterminée par simulation des courbes expérimentales selon la technique d'essais et erreurs. 


\section{RESULTATS.}

\subsection{Principe des mesures d'intensité.}

Après exposition et développement, on analyse la photographie (figure 3 ) avec un microdensitomètre de précision à balayage en $\mathrm{x}$ et y qui mesure la densité du noircissement.

L'enregistrement de l'intensité mesurée sur le diffractomètre avec le compteur proportionnel (fig 2) permet un repérage plus précis des angles, mais le rapport signal à bruit est plus faible que pour le film et ne permet pas de résoudre les oscillations secondaires observables sur le film. La comparaison, (figure.4), de la densité photographique et de l'intensité mesurée sur le diffractomètre montre un bon accord et indique que la densité du film est proportionnelle à la lumination (éclairement* temps de pose). La figure 5, comparant les densités de noircissement obtenues pour des temps de pose différents confirme ce résultat.

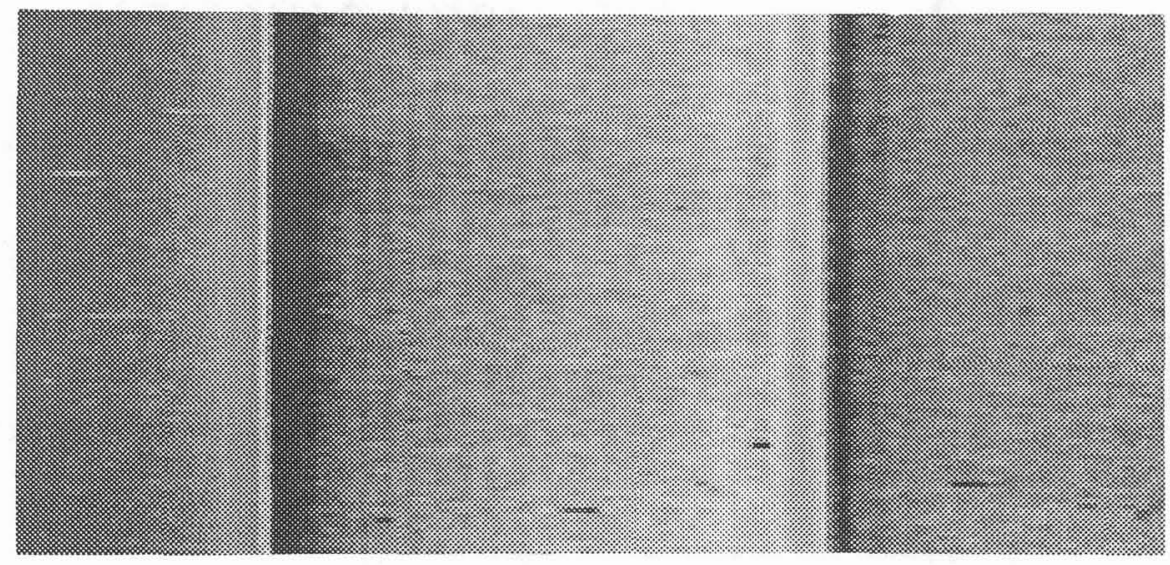

Figure 3: Fluorescence du fer dans une multicouche Fe/C périodique enregistrée en émergence rasante sur un film photographique.

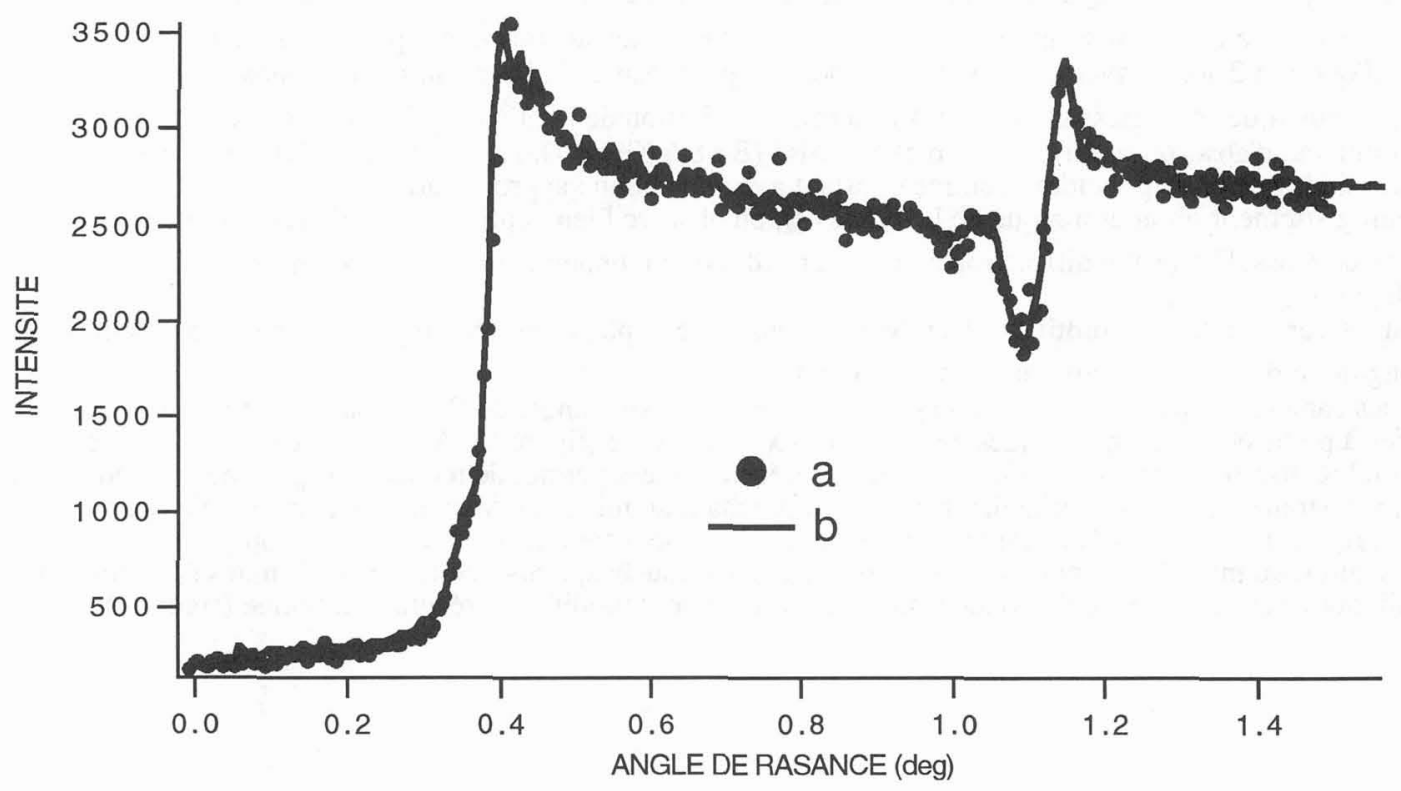

Figure 4: Comparaison de la répartition angulaire de l'intensité mesurée sur le diffractomètre avec le compteur proportionnel (a), et de la densité du film photographique, multipliée par une constante de normalisation (b). 


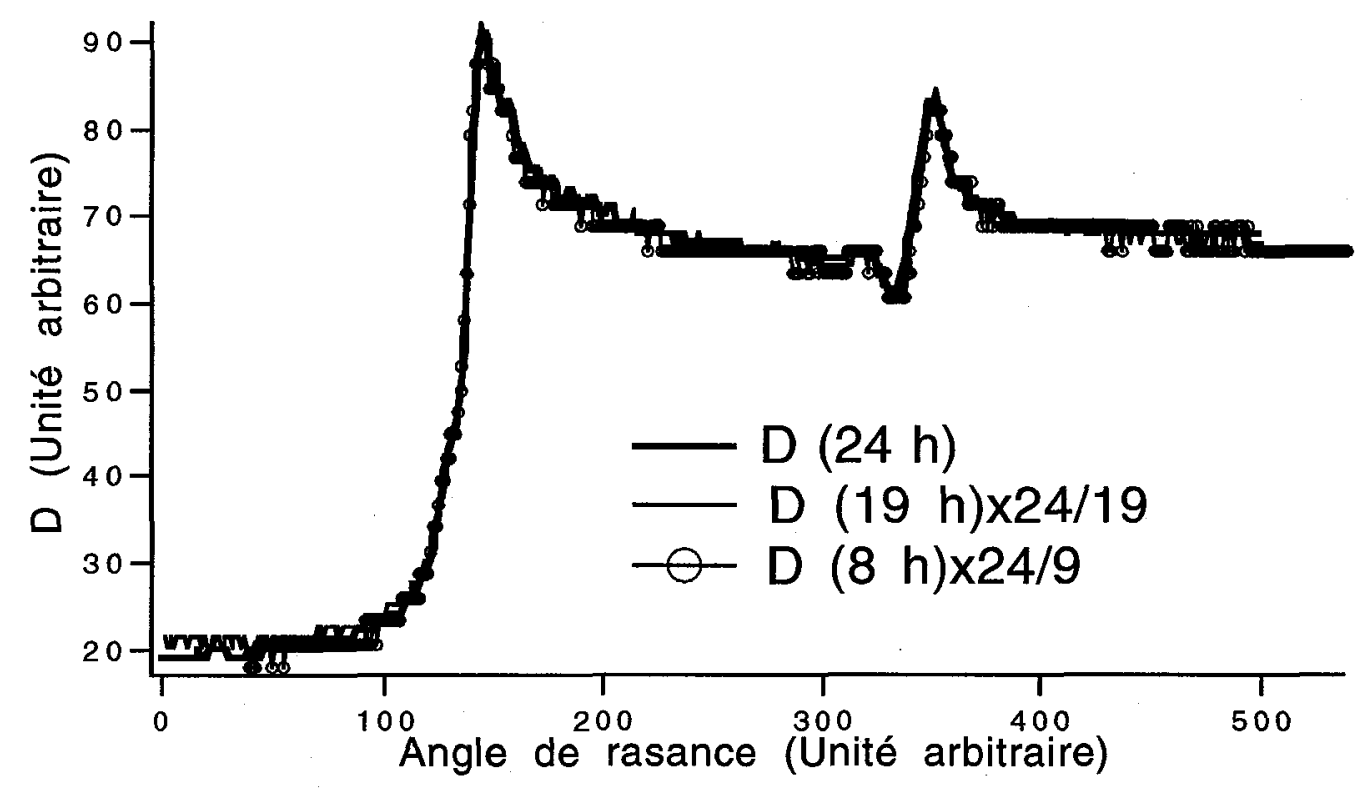

Figure 5: Linearité de la densité photographique en fonction de la lumination (Eclairement $x$ temps de pose) Les densités photographiques du même phénomene enregistré pendant des temps de pose différents sont proportionnelles au temps d'exposition dans une large gamme de lumination.

Les écarts entre les différentes courbes donnent une indication sur la précision des mesures obtenues par ce procédé.

\subsection{Fluorescence du Fer sous éclairage en incidence normale.}

Sous ces conditions d'éclairage, le champ électrique excitateur est pratiquement uniforme avec une décroissance lente due à l'absorption. Le calcul montre que l'intensité du champ pour la raie $\mathrm{K} \alpha$ du cuivre décroit d'environ $2 \%$ en traversant la multicouche. Il dépend peu de la longueur d'onde dans le domaine utile, correspondant à des énergies superieures à l'énergie d'excitation de la raie $\mathrm{K} \alpha$ du fer $(\mathrm{E}>6 \mathrm{Kev})$, et limitées par le domaine d'absorption du filtre de nickel utilisé $(E<8.5 \mathrm{Kev})$. Dans le cas d'un éclairage normal, on peut donc considérer le champ incident comme constant avec une bonne approximation.

Sur l'enregistrement photographique de la figure (3), on observe bien, après la zone de réflexion quasi-totale, la "ligne de Kossel" due à la diffraction au premier ordre du rayonnement $\mathrm{K} \alpha$ du fer par la multicouche . périodique [9].

La figure 6 représente (en pointillés), l'analyse en densité de la photographie du phénomène à travers un filtre de manganèse permettant d'éliminer la raie $K \beta$ du fer.

Quelques caractéristiques de l'empilement tels que: indice moyen, angle de Bragg, saut d'indice, peuvent être evaluées à partir de zones spécifiques de la courbe expérimentale (figure 6). A partir de ces premiers éléments, le logiciel réalisé selon le modèle de calcul décrit précedemment permet de rechercher, par une méthode essaierreur, les paramètres de la courbe théorique se rapprochant au mieux de la courbe expérimentale (trait continu, fig 6). Les écarts subsistant entre les deux courbes peuvent être attribués à une trop grande simplification du modèle de l'empilement, supposé à deux couches, alors que la fluctuation des épaisseurs dans la multicouche ou la présence de couches interfaciales peuvent modifier le résultat théorique (figure 7). 

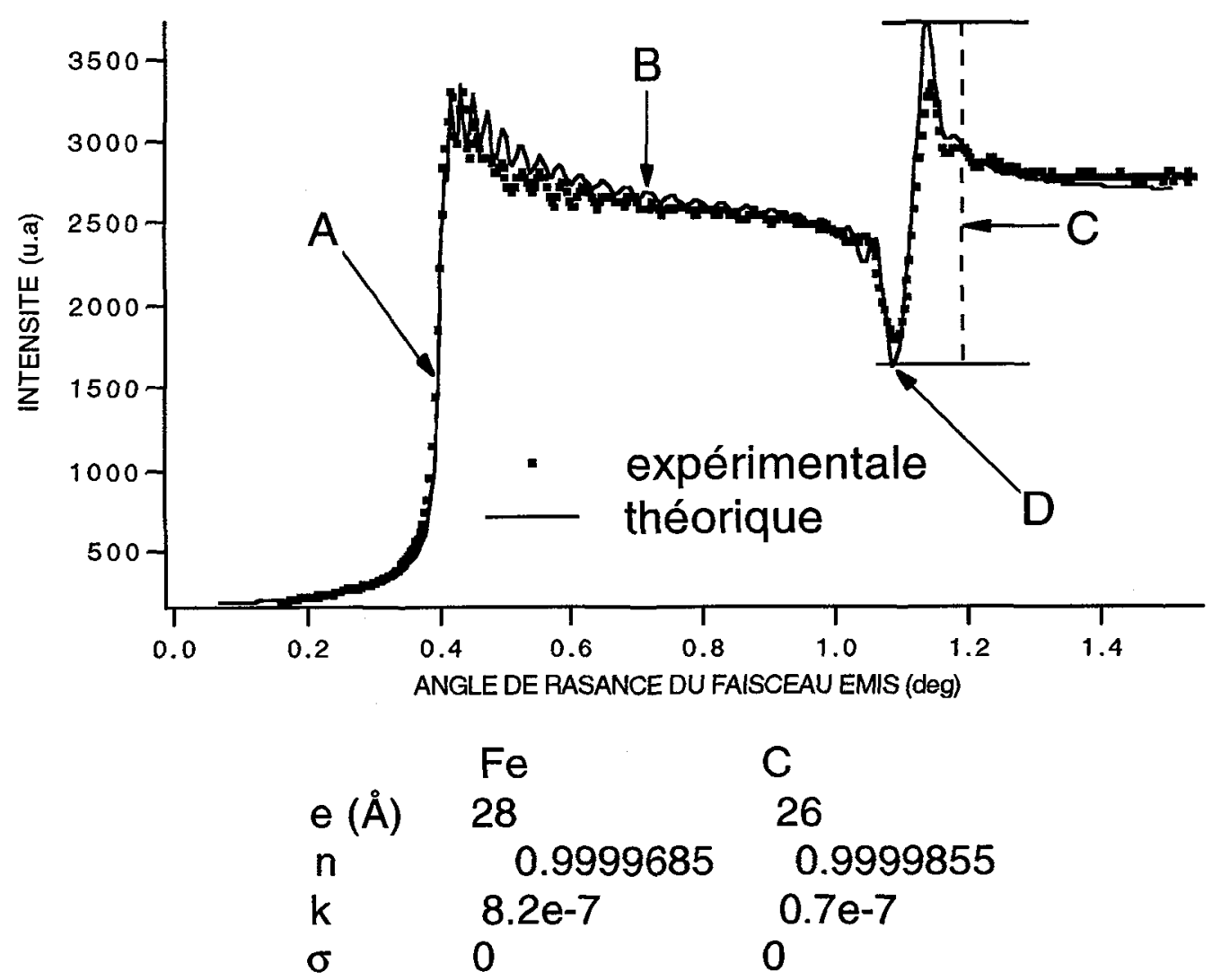

Figure 6 : Mesure de la répartition angulaire de l'intensitê de fluorescence du fer à travers un filtre de manganèse éliminant la raie $\mathrm{K}_{\beta}$ du fer (en pointillés). Les parties flêchées de la courbe experimentale correspondent à certaines caractéristiques de I'empilement : A: indice moyen, B: nombre de périodes, $\mathrm{C}$ : saut d'inđice, $\mathrm{D}$ : angle de Bragg. La courbe en trait continu a eté calculée à partir des valeurs indiquées dans le tableau ci-dessus.

En l'absence du filtre de manganèse, on obtient l'enregistrement (en pointillé) de la figure 8, montrant un pic atténué au voisinage de l'incidence de Bragg pour la raie $K \beta$ du fer, et un épaulement dû à cette même radiation, au voisinage de la zone de "réflexion totale". En calculant les intensités de fluorescence pour ces deux raies d'émission $K \alpha$ et $K \beta$ et en les ajustant avec le rapport de rendement approprie, on obtient la courbe en trait continu, représentative de la fluorescence pour l'ensemble de ces deux raies. 


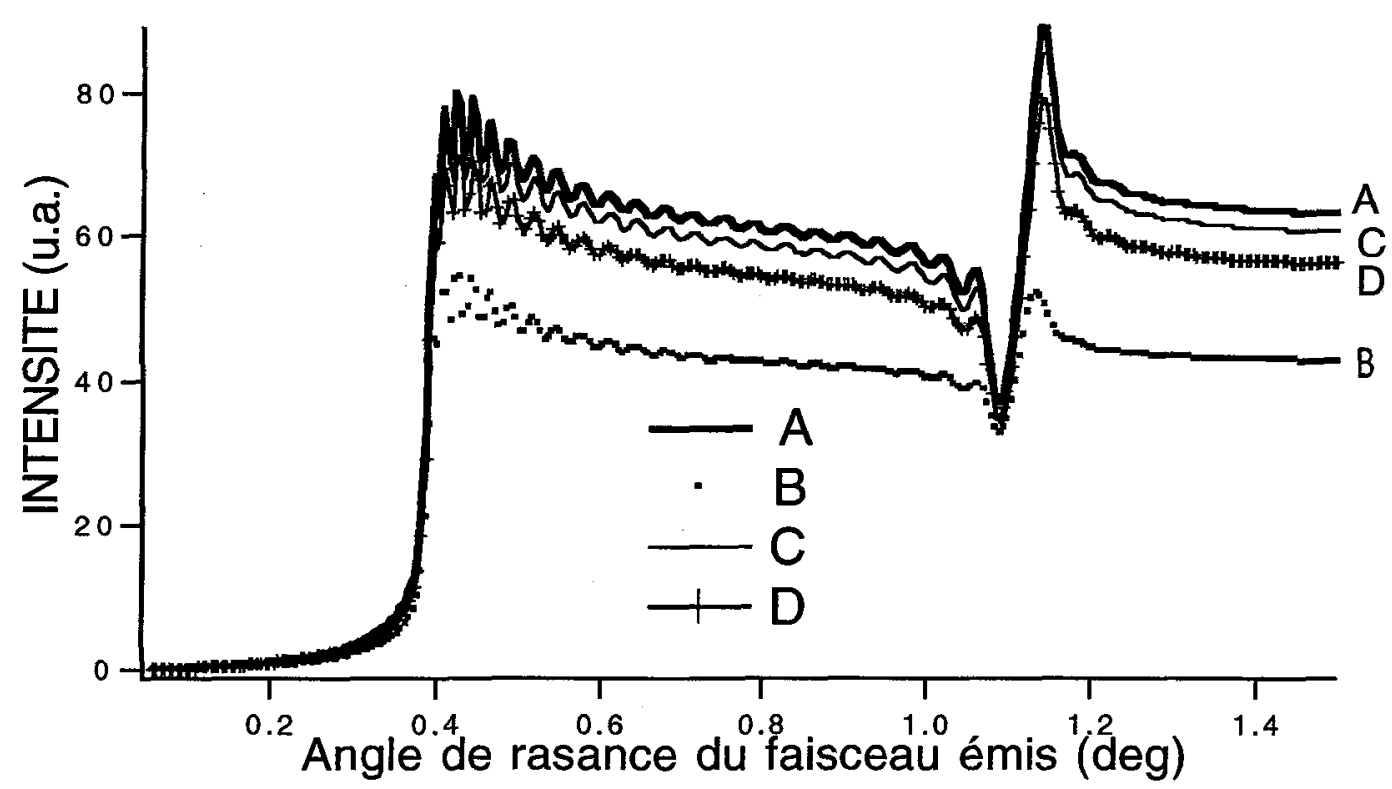

Figure 7: Dans le cas d'un éclairage normal, calcul de l'intensité de fluorescence de la raie $K_{\boldsymbol{\alpha}}$ du fer pour différents modèles de répartition des deux matériaux dans la bicouche elémentaire.. A- deux couches parfaites $\mathrm{Fe} / \mathrm{C}$; BInterdiffusion partielle homogene dans chacune des deux couches $(\mathrm{cFe}=0.8, \mathrm{c} C=0.2) ; \mathrm{C}$ - une couche interfaciale d'interdiffusiond'épaisseur $0.4 \mathrm{~nm}(\mathrm{cFe}=0.5, \mathrm{c} C=0.5)$; $\mathrm{D}$ - deux couches $\mathrm{d}^{\prime}$ interdiffusion d'épaisseurs respectives $0.4 \mathrm{~nm}$ et $0.8 \mathrm{~nm}\left(\mathrm{c}_{\mathrm{Fe}}=0.5, \mathrm{C} \mathrm{C}=0.5\right.$ ) situees respectivement aux interfaces $\mathrm{Fe} / \mathrm{C}$ et $\mathrm{C} / \mathrm{Fe}$.

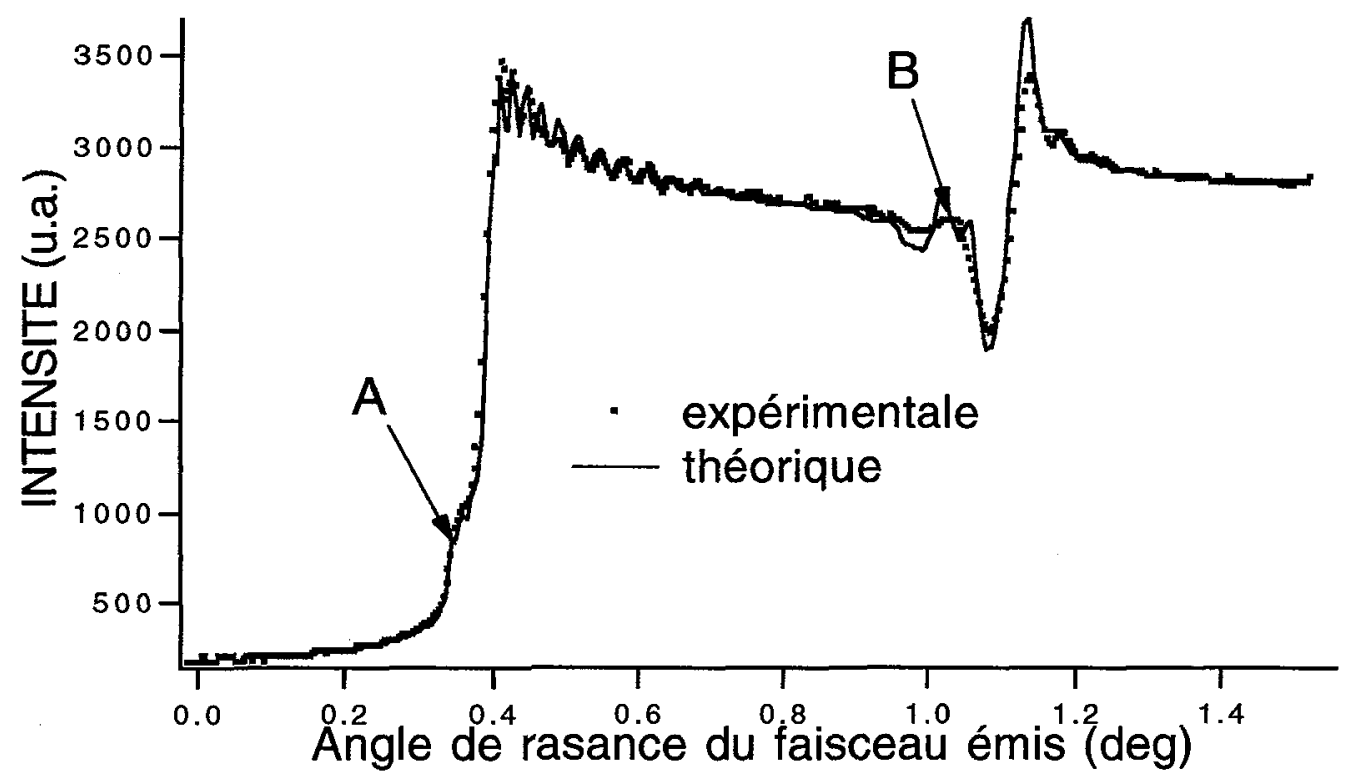

Figure 8: Répartition angulaire de l'intensité de fluorescence du fer en l'absence du filtre de Mn. Les zones A et B correspondent respectivement à la limite de réflexion totale pour la raie $\mathrm{K}_{\beta}$ du fer, et au maximum de la ligne de Kossel pour cette raie. La contribution à l'intensité totale, déterminé par la hauteur relative de la zone $\mathrm{A}$, est de 0.85 pour la raie $\mathrm{K}_{\alpha}$ et 0.15 pour $\mathrm{K}_{\beta}$. 


\subsection{Fluorescence du fer dans la multicouche sous incidence rasante.}

L'échantillon a été irradié avec le rayonnement de la source de cuivre du tube $\mathrm{X}$ du diffractomètre, la raie $\mathrm{K} \beta$ étant pratiquement éliminée par un filtre de nickel.

Le montage expérimental est légèrement différent du précédent; en effet, le film photographique doit être situé hors de la zone de réflexion du faisceau excitateur, et hors de la zone de diffusion répartie autour de ce faisceau. Le plan d'enregistrement est donc placé horizontalement au dessus du point d'impact du faisceau sur l'échantillon, parallèlement au plan d'indidence.

Le calcul montre que l'intensité du champ incident à l'intérieur de la multicouche varie considérablement avec l'angle d'attaque au voisinage de l'angle de Bragg. L'intensité de ce champ, à l'intérieur des premières couches de l'échantillon est représentée figure 9 . On constate que, suivant l'incidence, ce champ peut être plus ou moins intense dans la couche de fer, et on voit comment, par variation de l'angle d'attaque du faisceau incident, on peut exciter sélectivement les zones interfaciales dans la muticouche.

Les courbes théoriques de répartition angulaire des intensités d'émission de la raie $\mathrm{K} \alpha$ du fer présentent à la fois des différences de forme et d'intensité (fig 10) qui s'expliquent par ces différences de distribution du champ incident.

La figure 11 présente, dans le cas de l'incidence de Bragg, les courbes calculées pour différents modèles d'empilement dans la période, comparés au modèle idéal à deux couches. On peut noter que, dans le cas des deux couches interfaciales centrées respectivement sur les positions des maxima et minima du champ, seule la première d'entre elles contribue notablement à la fluorescence de la multicouche.

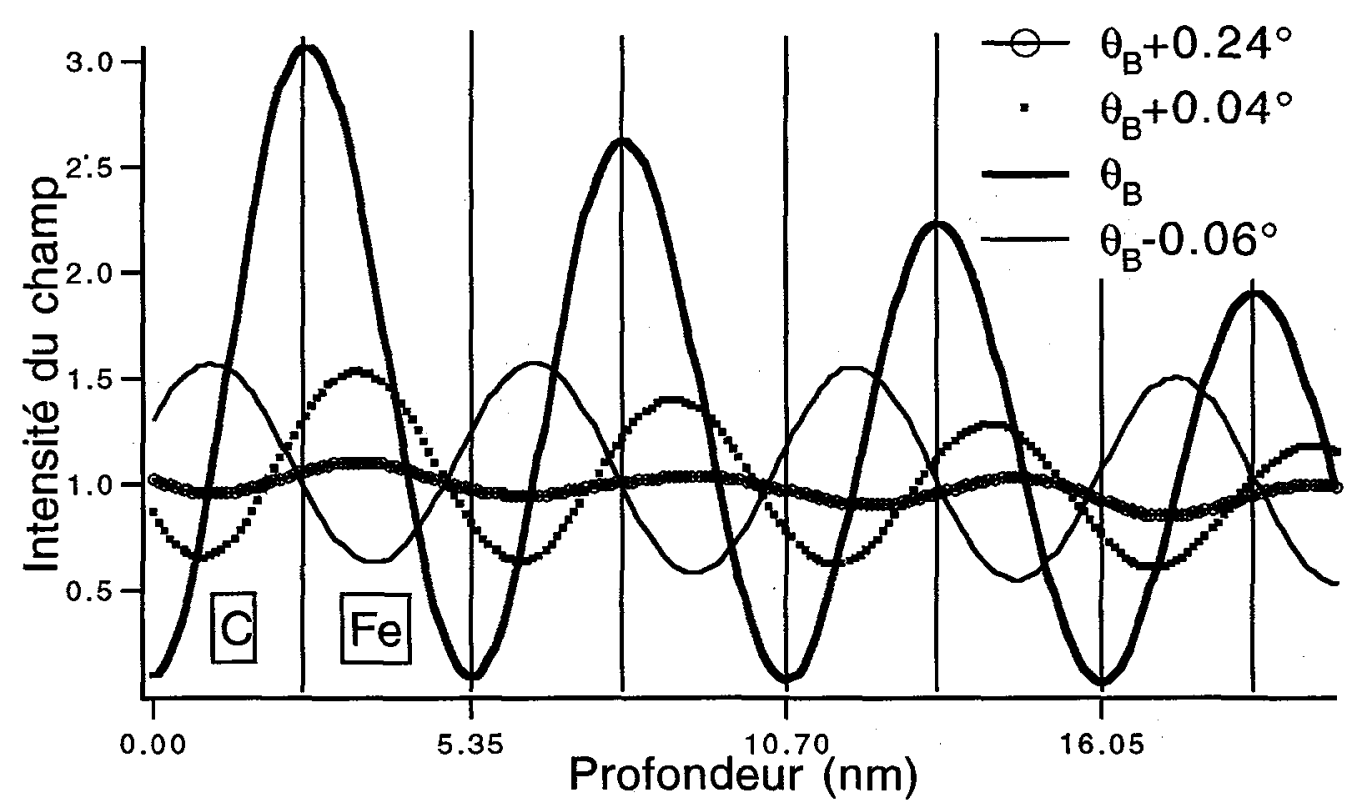

Figure 9: Intensité du champ excitateur ( $\mathrm{S}$ ou $\mathrm{P}$ ) dans la multicouche Fe/C sous éclairage rasant au voisinage de l'angle de Bragg $\theta_{B}$ (on indique les angles de rasance). 


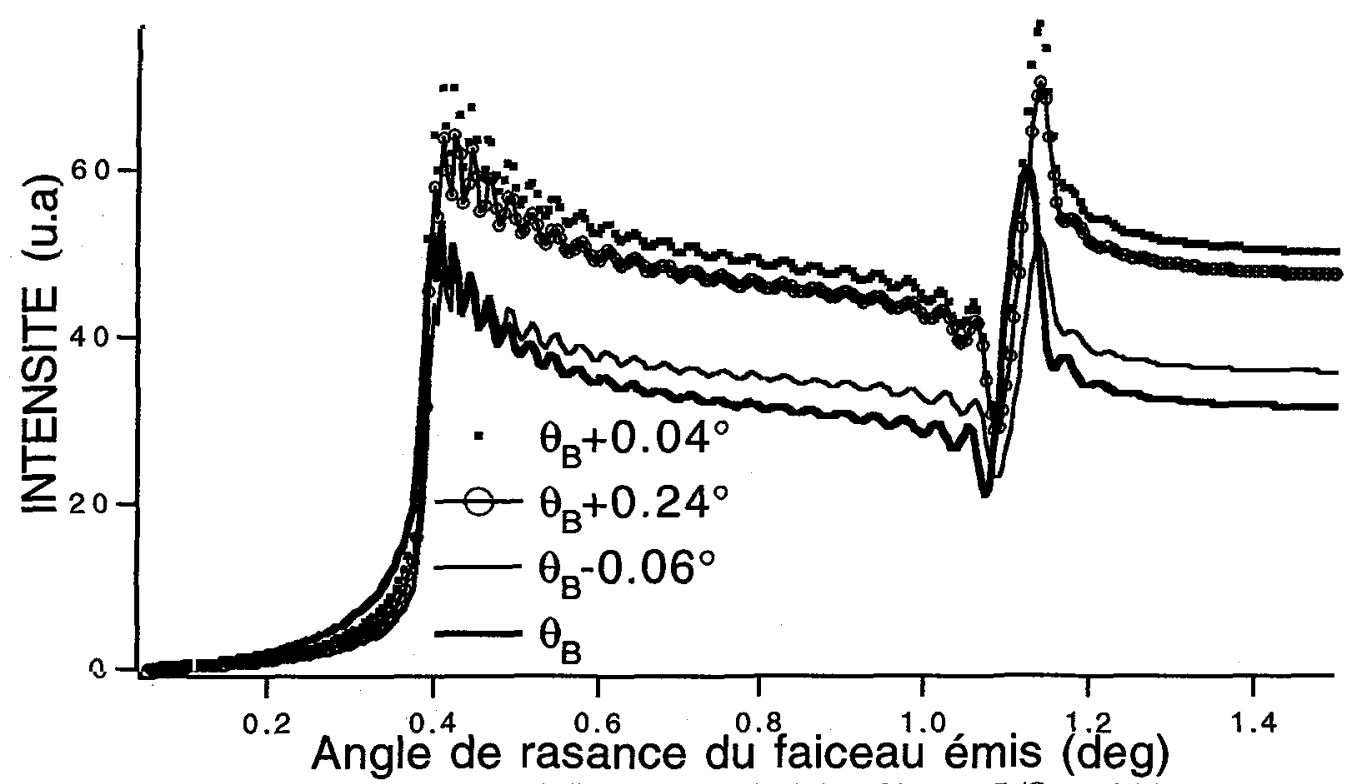

Figure 10: Intensité calculee du rayonnement de fluorescence du fer de la multicouche $\mathrm{Fe} / \mathrm{C}$ sous éclairage rasant, au voisinage de l'angle de Bragg.

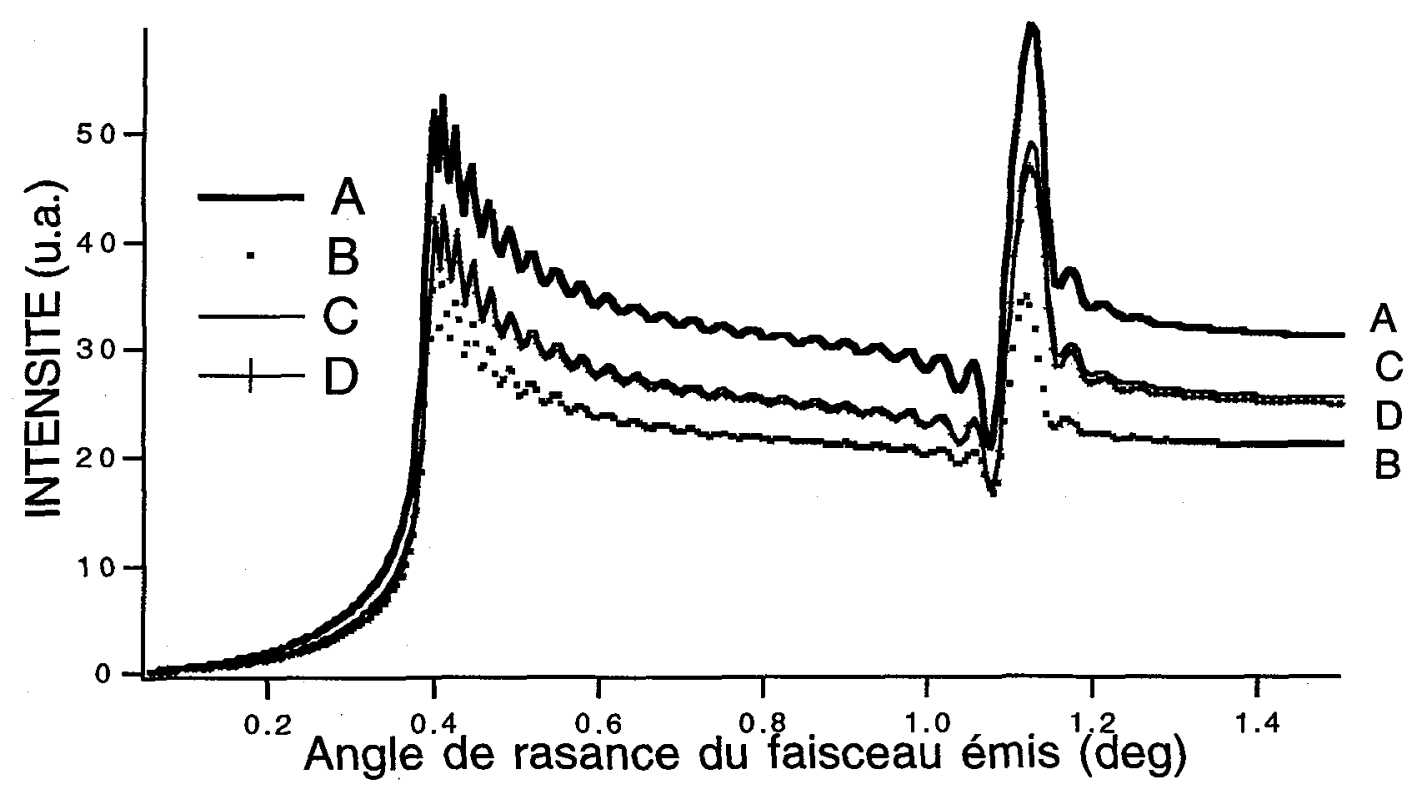

Figure 11: Calcul de l'intensité de fluorescence de la raie $\mathrm{K}_{\alpha}$ du fer pour différents modeles de répartition des deux matériaux dans la bicouche élémentaire, dans le cas d'un éclairage rasant sous l'angle de Bragg.. A- Deux couches parfaites $\mathrm{Fe} / \mathrm{C}$; B-Interdiffusion partielle homogène dans chacune des deux couches $\left(\mathrm{cFe}=0.8, c_{C}=0.2\right)$; $\mathrm{C}$ - une couche interfaciale d'interdiffusion d'épaisseur $0.4 \mathrm{~nm}\left(\mathrm{cFe}_{\mathrm{F}}=0.5, \mathrm{cC}=0.5\right)$ centré au point où l'intensite du champ incident est maximum; $D$ - en plus de la couche $C$, une couche interfaciale d'épaisseur $0.8 \mathrm{~nm}\left(\mathrm{CFe}_{\mathrm{C}}=0.5, \mathrm{c} C=0.5\right)$, située autour $\mathrm{d}^{\prime}$ un minimum du champ excitateur. 

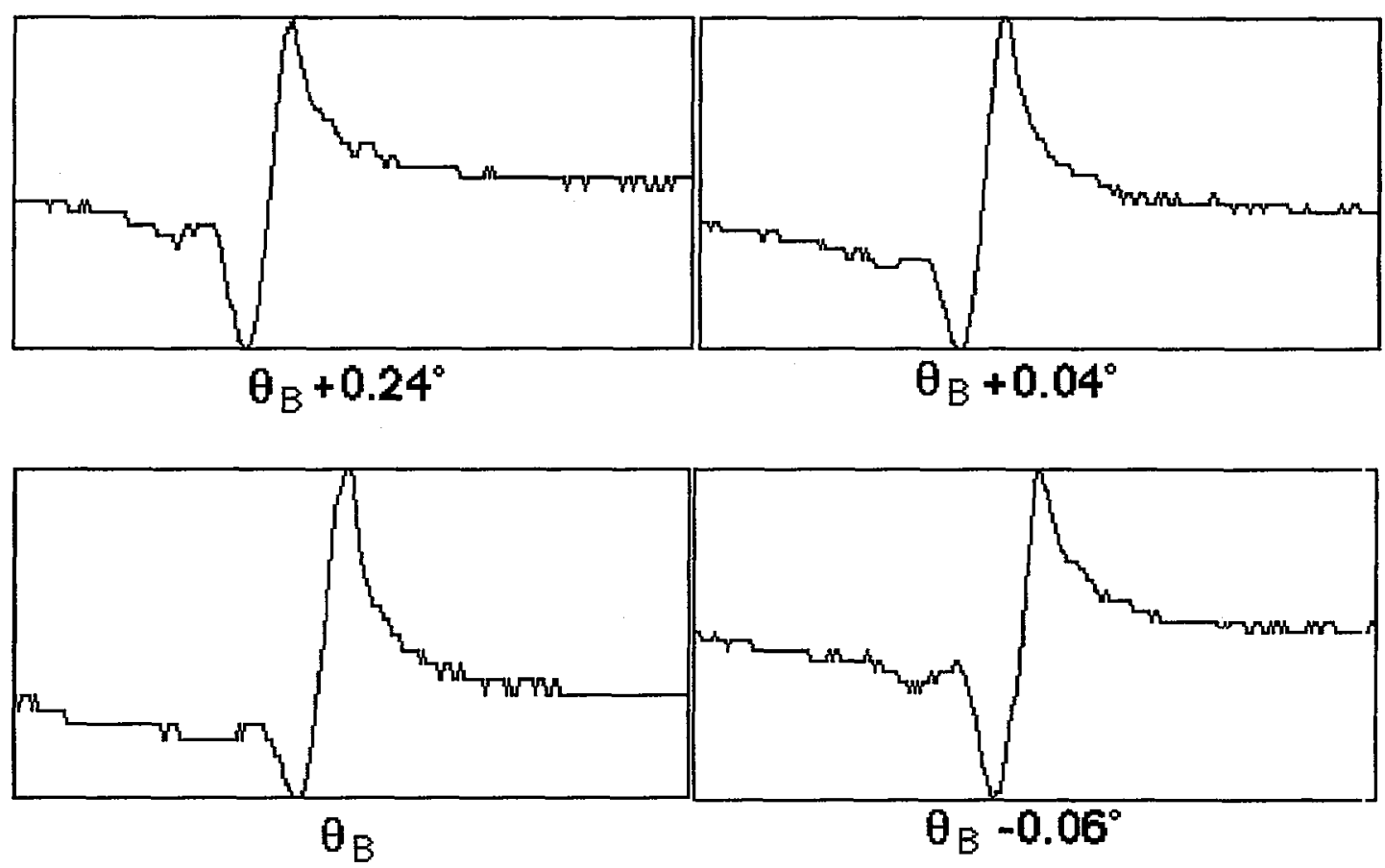

Figure 12: Lignes de Kossel expérimentales obtenues sous différents angles d'éclairage rasant, au voisinage de l'angle de Bragg $\theta_{B}$.

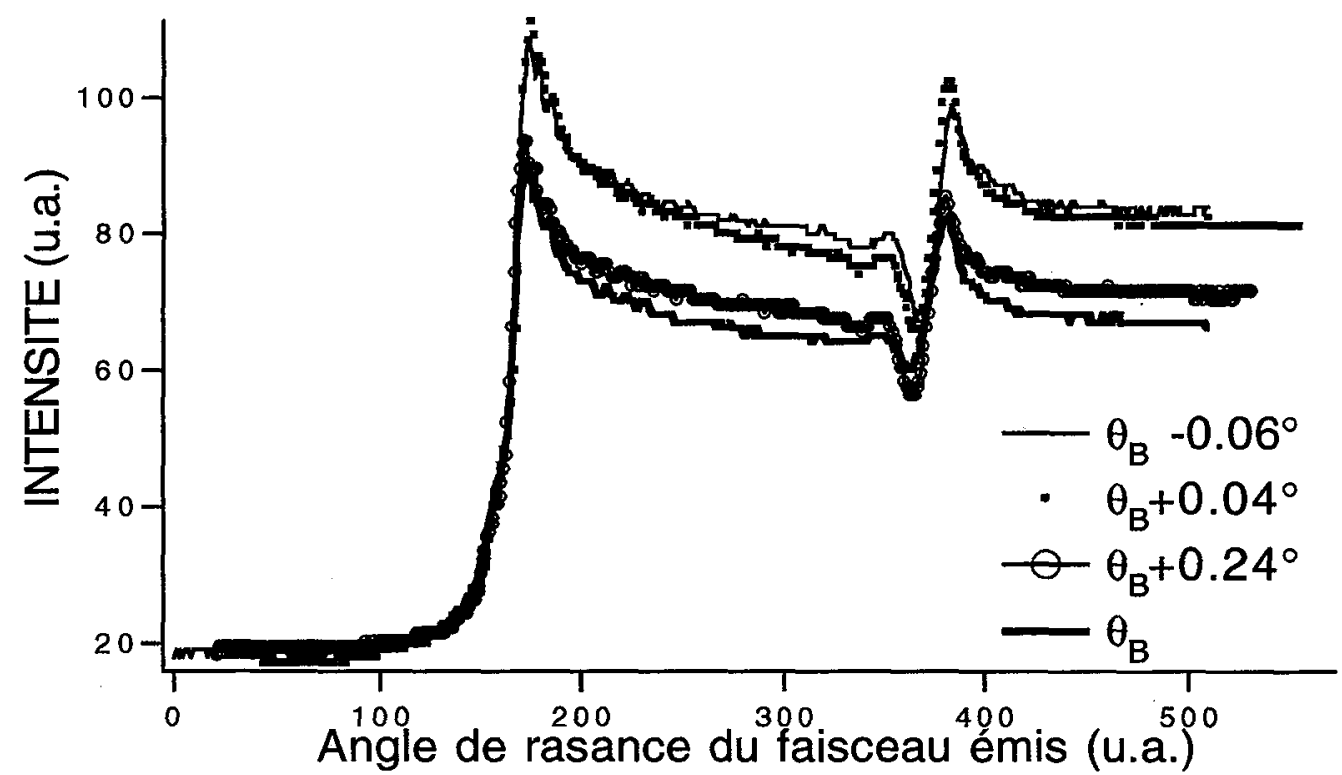

Figure 13: Mesures de l'intensité de fluorescence du fer sous différents angles de rasance du faisceau d'éclairage, au voisinage de l'angle de Bragg. $\theta_{\mathrm{B}}$.

Les résultats expérimentaux de la figure (12) montrent les lignes de Kossel obenues pour différents angles de rasance, et peuvent être comparés aux résultats théoriques de la figure (10). On remarque l'aspect caractéristique de la courbe obtenue sous éclairage en incidence de Bragg, montrant une dissymétrie 
importante entre le maximum et le minimum d'intensité, par rapport à la valeur moyenne, hors de la zone de l'angle de Bragg.

La figure 13 présente les intensités comparées des différentes mesures. Des différences apparaissent,avec les résultats théoriques de la figure 10, élaborés à partir d'un modèle simple d'empilement. Une analyse plus poussée est nécessaire en supposant par exemple la formation de couches d'interdiffusion dans les régions interfaciales.

\section{CONCLUSION.}

En utilisant les principaux éléments d'un diffractomètre à rayons $\mathrm{X}$ classique, nous avons mesuré les variations angulaires du rayonnement de fluorescence $\mathrm{K} \alpha \mathrm{du}$ fer émis par une multicouche périodique Fe/C, et montré qu'elles dépendaient notablement de l'angle d'incidence du rayonnement excitateur dans. le voisinage de l'angle de Bragg du premier ordre de la multicouche. Une modélisation de l'intensité de fluorescence émise par une multicouche supposée parfaite et réalisée en utilisant les méthodes de calcul des propriétés optiques des multicouches, sur la base du théorème de réciprocité, montre que cette évolution est provoquée par le déplacement rapide des extrêma de l'onde excitatrice quasi-stationnaire par rapport aux interfaces dans l'empilement, lorsque l'incidence varie autour de l'angle de Bragg.

Il reste à prendre en compte les défauts de la multicouche pour obtenir une simulation quantitative des courbes expérimentales. Cette technique non-destructive devrait permettre d'affiner la détermination du profil de concentration des matériaux dans les multicouches, en complément de la réflectométrie de rayons $\mathrm{X}$ rasants.

\section{Références.}

[1] Spiller E., Soft X-ray Optics, (SPIE Opt. Eng. Press, Bellingham, Washington, 1994) pour une bibliographie détaillée sur ce sujet.

[2] Névot L., Pardo B., Corno J., Revue Phys. Appl., 23, (1988), 1675-1686.

[3] Brunel M., de Bergevin F., Acta Cryst., A42, (1986), 299-309.

[4] de Boer D. K. G., Phys. Rev. B, 44, (1991), 498-511.

[5] Kossel W., Loeck V., Voges H.; Zeit. f. Physik; 94; (1935); 139.

[6] Becker R.S., Golovchenko J.A., Patel J.R., Phys. Rev. Lett. 50, (1983), 153-156.

[7] Sasaki Y.C., Hirokawa K., Appl. Phys. Lett., 58, (1991), 1384-1386.

[8] Noma T., Iida A., Sakurai K., Phys. Rev. B, 48,(1993), 17524-17526.

[9] Chauvineau J.P., Bridou F., Hainaut O., J. Phys. IV, (1996) A paraître.

[10] Bridou F., Pardo B.A., J. Optics, 21, (1990), 183-191.

[11] Bridou F., J. Phys., 4, (1994), 1513-1522.

[12] Berning P. H., Physics of thin films, 1, (G. Hass, Academic Press, 1963), 69.

[13]. Lorentz H.A.; Proc. Amsterdam Acad.; 8; (1905); 401.

[14]. v. Laue M.; Ann. der Physik; 23; (1935), 705.

[15]. Henke B.L., Gullikson E.M., Davis J.C., Atomic Data and Nucl. Tables, 54, (1993), 181-3424. 